PSI-PR-95-09

IPS Report 95-14

UCY-PHY-95/5

\title{
Full QCD with the Lüscher local bosonic action
}

\author{
C.Alexandrou ${ }^{1,2}$, A. Borrelli ${ }^{2}$, Ph. de Forcrand ${ }^{3}$, A. Galli ${ }^{2}$, F. Jegerlehner ${ }^{2}$ \\ 1 Department of Natural Sciences, University of Cyprus, CY-1678 Nicosia, Cyprus \\ 2 Paul Scherrer Institute, CH-5232 Villigen, Switzerland \\ 3 IPS, ETH-Zentrum, CH-8092 Zürich, Switzerland
}

\begin{abstract}
We investigate Lüscher's method of including dynamical Wilson fermions in a lattice simulation of QCD with two quark flavours. We measure the accuracy of the approximation by comparing it with Hybrid Monte Carlo results for gauge plaquette and Wilson loops. We also introduce an additional global Metropolis step in the update. We show that the complexity of Lüscher's algorithm compares favourably with that of the Hybrid Monte Carlo.
\end{abstract}




\section{Introduction}

Taking into account the effects of sea-quarks in lattice QCD simulations presents serious technical difficulties. The main problem is the excessive cost in computer time due to the need of evaluating the fermion matrix determinant for each gauge field update step. The most used algorithm, Hybrid Monte Carlo (HMC) [1], encounters dramatic problems of CPU time costs and slowing down in the limit of small quark masses, a fact that has generally forced people to use the valence (quenched) approximation in which the fermion determinant is assumed to be unity. For many observables the results obtained in this approximation fit quite well with phenomenologically known properties of QCD, but for other observables the quenched approximation is definitely too crude (for example $\alpha_{s}$ ).

The urgent need to be able to perform calculations in full QCD has motivated substantial activity to overcome the technical problems caused by the full dynamical treatment of the fermions. The search for a less expensive algorithm for lattice sea-quarks simulation is therefore a very actual subject, on which recently a new proposal has been made by M. Lüscher [2]. The new method consists in approximating the inverse of the fermion matrix with a polynomial, and then interpreting the determinant as the partition function of a local bosonic system. Simulating this bosonic system will enable us to evaluate the effects of the sea-quarks.

This algorithm is quite general, and its application to various systems has been and is being studied, with the aim of optimizing it and rendering it economically viable. In this paper, we will report the results of a first exploratory application of Lüscher's algorithm to an SU(3) Lagrangian with two degenerate flavours of Wilson quarks. The observables measured are the gauge plaquette and Wilson loops, for which we study the autocorrelation time and precision of the approximation for numerous sets of parameters. We also show the effect of inserting a global Metropolis step in the update, as suggested in reference [3], with the aim of making the algorithm more precise and more efficient.

\section{The algorithm}

The partition function of lattice QCD with two fermion flavours can be written as

$$
Z=\int[d U] \operatorname{det} Q^{2} e^{-S_{G}[U]}
$$

where $S_{G}[U]$ is the pure gauge action and the fermion matrix $Q$ has the form:

$$
Q=\frac{\gamma_{5}(D+m)}{c_{M} M}, \quad \text { with } \quad M=\frac{8}{a}+m .
$$

Here $a$ is the lattice spacing, and $Q$ has been chosen hermitian, and with eigenvalues contained in the interval $(-1,1)$. The constant $c_{M} \geq 1$ is fixed to the values 1.1 as explained in ref. [2].

As anticipated, the first step is approximating $1 / Q^{2}$ with a polynomial $P\left(Q^{2}\right)$. In choosing the form of $P(s)$, we follow [2], where its explicit expression in terms of Chebyshev polynomials is given. 
$P(s)$ is of even degree $n$, and approximates the function $1 / s$ in an interval $\epsilon \leq s \leq 1$, where $\epsilon$ is a parameter to be chosen according to the range of eigenvalues of $Q^{2}$; the $n$ roots of $P(s), z_{k}$, lie on an ellipse in the complex plane with foci $\epsilon$ and 1 , and minor axis of length $2 \sqrt{\epsilon}$; they come in complex conjugate pairs, with $\operatorname{Im} z_{k} \neq 0$. Since the roots come in complex conjugate pairs, $P(s)$ can be written in the form:

$$
P(s)=\text { const } \times \prod_{i=1}^{n}\left(s-z_{k}\right)=\text { const } \times \prod_{i=1}^{n}\left(\sqrt{s}-\sqrt{\bar{z}_{k}}\right)\left(\sqrt{s}-\sqrt{z_{k}}\right) .
$$

A measure of the relative error of the approximation is given by $R(s)=P(s) s-1$, which in the interval $\epsilon \leq s \leq 1$ is bounded by

$$
|R(s)| \leq 2\left(\frac{1-\sqrt{\epsilon}}{1+\sqrt{\epsilon}}\right)^{n+1} \equiv \delta(n, \epsilon) .
$$

When $s<\epsilon$ the polynomial continues to converge, but with an $s$-dependent exponential rate approaching zero in the $s \rightarrow 0$ limit.

We can now introduce a set of boson fields $\phi_{k}(k=1, \ldots n)$ with partition function $Z_{b}$, approximating the fermion determinant

$$
Z_{b}[U]=\int \prod_{k=1}^{n}\left[d \phi_{k}\right]\left[d \phi_{k}^{\dagger}\right] e^{-\phi_{k}^{\dagger}\left(Q-\sqrt{\overline{z_{k}}}\right)\left(Q-\sqrt{z_{k}}\right) \phi_{k}}=\frac{1}{\operatorname{det} P\left(Q^{2}\right)} \simeq \operatorname{det} Q^{2} .
$$

The partition function of full QCD is then approximated by the local bosonic action

$$
Z=\int[d U] \operatorname{det} Q^{2} e^{-S_{G}[U]} \simeq \int[d U] Z_{b}[U] e^{-S_{G}[U]}
$$

Making use of this action, we may now simulate the fermion determinant by locally updating the boson fields $U$ and $\phi$, using algorithms like heat-bath and over-relaxation. The implementation of the program is described in Appendix A.

\section{$3 \quad$ Numerical simulations on the $4^{4}$ lattice for the pure Lüscher algorithm}

The numerical simulations of the theory described by (6) were performed on a $4^{4}$ lattice for various choices of the parameters $\epsilon$ and $n$, at $\beta=6.0$, for $\kappa=0.12$ and 0.14 (in the deconfined phase), and at $\beta=5.0$ for $\kappa=0.15$ (in the confined phase).

For each set of parameters, an average of 10,000 sweeps were performed, each sweep consisting of one $\phi$ heat-bath, one $\phi$ over-relaxation, and one to six $U$ over-relaxations (see further on for discussion), ergodicity being ensured by the $\phi$ heat-bath. After each sweep the gauge boson plaquette was measured.

For all $\kappa$ values, the minimum eigenvalue of $Q^{2}$ was measured for approximately 10,000 configurations. The average results are:

$$
\begin{array}{ll}
0.015 \pm 0.001 & (\kappa=0.12, \beta=6) \\
0.006 \pm 0.001 & (\kappa=0.14, \beta=6) \\
0.003 \pm 0.001 & (\kappa=0.15, \beta=5)
\end{array}
$$


The averages of the lowest eigenvalues are shown in Fig. 1 for $\kappa=0.12$ and $\beta=6$. As a check, measurements of the plaquette with different numbers of $U$ field over-relaxation steps have been done, monitoring the autocorrelation time: no variation was observed, indicating that the autocorrelation time was dominated by the dynamics of the $\phi$ fields. Consequently we chose to perform in subsequent runs only one $U$ over-relaxation per sweep.

For comparison, the plaquette was measured for the same lattice parameters and $\kappa$ values also using a HMC program. The results of all the simulations are reported in Table 1. In Fig. 2 the plaquette values are plotted as a function of $\delta(n, \epsilon)$.

As can be seen from Table 1, many values of $\epsilon$ chosen are quite high with respect to the lowest $Q^{2}$ eigenvalue; despite that, the agreement with the HMC results remains good, on this $4^{4}$ lattice, up to $\epsilon$ values ten times larger than the lowest $Q^{2}$ eigenvalue. This shows that the first few low-energy modes of the fermion determinant do not couple strongly to the plaquette. On larger lattices where non-local observables can be measured better, one would expect that $\epsilon$ will have to be chosen closer to the lowest eigenvalue.

It is also interesting to observe that the convergence of the plaquette to its correct value is not exponential in $n$; in fact it is not necessarily monotonic. For $\beta=5$. for example it approaches the correct value from above, while the quenched $(n=0)$ value is below the full QCD one. This unexpected behaviour occurs when $\epsilon$ is taken much larger then $\lambda_{\min }\left(Q^{2}\right)$. In such cases $\delta$, taken alone, can not be a good measure of the error: The bulk of the error comes from small eigenvalues $\lambda<\epsilon$.

For $\kappa=0.12$, the results show that we can push $\epsilon$ up to 0.1 , while we see a definite breakdown of the approximation at $\epsilon=0.5, \quad n=4\left(\delta \sim 2 . \times 10^{-4}\right)$. As we approach more critical $k$ values, the eigenvalues of $Q^{2}$ decrease and, for $\kappa=0.14$, we see that the approximation breaks down already at $\epsilon=0.08, \quad n=16,\left(\delta \sim 1 . \times 10^{-4}\right)$.

For $\beta=5$ and $\kappa=0.15$, in the confined phase, the minimum eigenvalue is $\sim 0.003$, and satisfactory results can be obtained at $\epsilon=0.01, n=50,\left(\delta \sim 7 \times 10^{-5}\right)$.

Since the CPU costs are expected to be at least proportional to the number of fields $n$, and, in turn, a smaller value of $\epsilon$ requires a larger $n$, we conclude that the most economical program will use the highest $\epsilon$ and lowest $n$ values compatible with a good approximation of the fermion determinant. A more detailed discussion of the CPU costs for obtaining a set of uncorrelated configurations is found in the next section. From our observation of the plaquette on a $4^{4}$ lattice, it appears that one can raise $\epsilon$ up to values significantly larger than the lowest $Q^{2}$ eigenvalue.

It will be shown in section 5 , that the situation can be further improved by the insertion of a global Metropolis test in the updating procedure for the $\phi$ and $U$ fields. The modified method will allow us to maintain a good approximation for higher $\epsilon$ and therefore lower $n$, thus reducing the CPU cost of the simulation. 


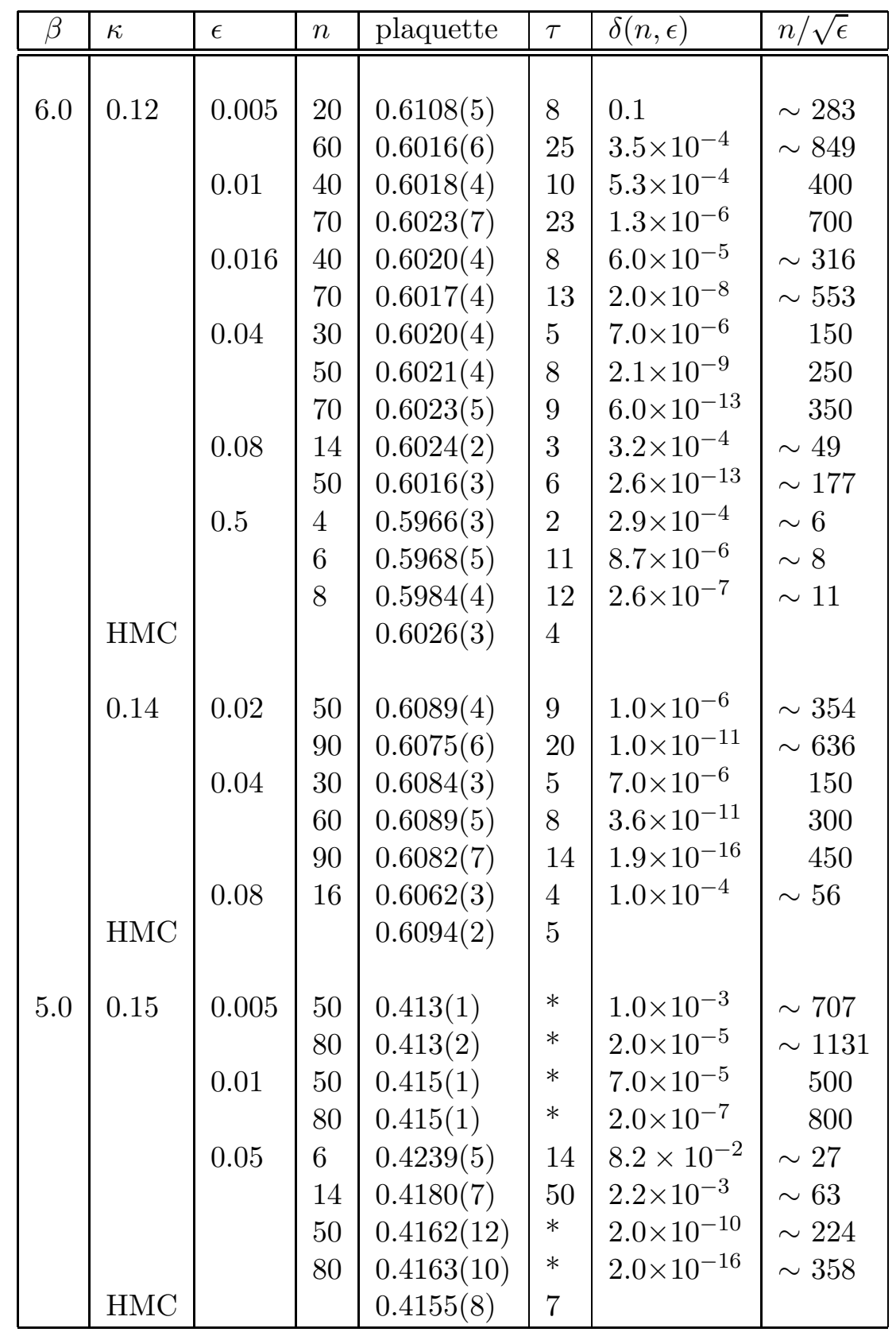

Table 1: Simulation results for the plaquette on a $4^{4}$ lattice at $\beta=6$ for the Lüscher algorithm and the HMC algorithm. Each plaquette value is an average of about $10^{\prime} 000$ measurements. A star * means that the autocorrelation time was too long to be measured accurately. The autocorrelation time of the Lüscher algorithm is measured in unit of iteration sweeps, consisting of one $\mathrm{HB}$ and OR of the $\phi$ and one OR of the $U$. The autocorrelation time of the HMC is measured in unit of trajectories, each of them consisting of 12 molecular dynamic steps. 


\section{Autocorrelation and scaling behaviour for the pure Lüscher algorithm}

We have measured the autocorrelation of the plaquette in the deconfined and confined phases. In Fig. 3, the plaquette autocorrelation time $\tau$ is shown versus the ratio $\frac{n}{\sqrt{\epsilon}}$, in the deconfined phase: the relationship between the two appears to be linear. On the other hand, if we freeze the bosonic fields $\phi$, the autocorrelation time of the plaquette is $\mathcal{O}(1)$ sweep of the gauge fields. So the long correlation times are caused by the slow dynamics of the $\phi$ fields. These bosons are updated by local algorithms (e.g. heat-bath) which have a critical dynamical exponent of 2 in the worst case scenario. This means that, if the correlation length of the bosonic action is $\xi$ in lattice units, then the correlation time will be $\sim \xi^{2}$. What then is the correlation length ? The bosonic action is of the form $\left|\left(Q-\mu_{k}\right) \phi_{k}\right|^{2}+\left|\nu_{k} \phi_{k}\right|^{2}=\left|\left(\gamma_{5} Q-\gamma_{5} \mu_{k}\right) \phi_{k}\right|^{2}+\left|\nu_{k} \phi_{k}\right|^{2}$. In the deconfined phase the Dirac matrix $\gamma_{5} Q$ is chirally symmetric. But the second term $\gamma_{5} \mu_{k}$ is not: it breaks chiral symmetry, with a characteristic length scale $1 / \mu_{k}$. Therefore we expect that the smallest mass term $\mu=\min _{k}\left(\mu_{k}\right)$ will control the autocorrelation time: $\tau \sim \xi^{2} \sim \frac{1}{\mu^{2}}$. It is easy to observe from the definition of the roots that the smallest $\mu^{-2}$ is proportional to $n / \sqrt{\epsilon}$. The same conclusion $\tau \sim n / \sqrt{\epsilon}$ can be reached also by considering the correlation length $1 / \nu_{k}$ given by the third term of the bosonic action above [2].

In the confined phase, an additional length scale $1 / m_{\pi}$ appears because of chiral symmetry breaking. This length scale may obscure the previous relationship $\tau \sim 1 / \sqrt{\epsilon}$, and weaken the dependence of $\tau$ on $\epsilon$. Unfortunately our numerical simulations are not accurate enough to resolve this issue: they only indicate that $\tau$ increases very much in the confined regime, for a given quark mass and number of bosonic fields.

While the cost in memory of the program is proportional to the volume of the lattice and to the number of auxiliary boson fields, the work (or CPU time) necessary to obtain two uncorrelated configurations is proportional to the number of boson fields times the autocorrelation time $\tau$.

Assuming the same $1 / \sqrt{\epsilon}$ dependence of $\tau$ in both phases, which should be a worst case scenario, we can predict the slowing down of the algorithm as the quark mass $m_{q}$ decreases. $\epsilon$ must be tuned according to the lowest eigenvalue of the $Q^{2}$ matrix and is therefore proportional to $m_{q}^{2}$. Requiring the error $\delta$ to be constant one obtains from (4) that $n \sim 1 / m_{q}$ and therefore, for a fixed volume, the autocorrelation time increases like $m_{q}^{-2}$. Since the work per sweep is proportional to the number $n \sim 1 / m_{q}$ of fields to update, one finally obtains that the work to obtain a new, decorrelated configuration, scales like $m_{q}^{-3}$.

As the volume $V$ of the lattice is increased, the scaling behaviour of the algorithm can be easily predicted from the exponential convergence of the approximation eq. (函). Let us keep the quark mass, and thus $\epsilon \sim m_{q}^{-2}$, fixed. Then requiring that the accumulated error of the approximation remain constant as $V$ is increased leads to $n \sim \log V$. The autocorrelation time is proportional to $n$, and the work to obtain a new, decorrelated configuration proportional to $n^{2}$, so that the work scales like $V(\log V)^{2}$.

To summarize this section, the needed work per new configuration is at most propor- 
tional to $V(\log V)^{2} m_{q}^{-3}$. This compares favourably with that of the HMC algorithm, $V^{5 / 4} m_{q}^{-7 / 2}$ or $V^{5 / 4} m_{q}^{-13 / 4}$, depending on the scaling of the trajectory length with $m_{q}$ [6]. Either way, the Lüscher algorithm is asymptotically faster, with respect to both large volumes and small quark masses.

\section{The systematic error and the exact algorithm}

The exact partition function of QCD is given by

$$
\begin{aligned}
Z & =\int[d U] \frac{\operatorname{det} Q^{2} \operatorname{det} P\left(Q^{2}\right)}{\operatorname{det} P\left(Q^{2}\right)} e^{-S_{G}[U]} \\
& =\int[d U] \operatorname{det}\left(Q^{2} P\left(Q^{2}\right)\right) Z_{b}[U] e^{-S_{G}[U]} .
\end{aligned}
$$

The partition function used in eq. (6) corresponds to approximating $\operatorname{det}\left(Q^{2} P\left(Q^{2}\right)\right)$ with unity. Hence, for a given configuration of gauge fields $U$, the error involved in the approximation of the determinant is given by

$$
R[U]=\operatorname{det} Q^{2} P\left(Q^{2}\right)-1=\prod_{i} \lambda_{i} P\left(\lambda_{i}\right)-1
$$

where the last step follows by simultaneously diagonalising $P\left(Q^{2}\right)$ and $Q . \lambda_{i}$ are the eigenvalues of $Q^{2}$ for the configuration $U$.

The actual value of this systematic error changes between two different configurations $U$ and $U^{\prime}$ as $R[U]$ does. The amplitude of these fluctuations can be tuned by an appropriate choice of the approximation parameters, the polynomial degree $n$ and the cut-off $\epsilon$. Of course, the cost in memory and CPU time will dramatically increase if we are trying to improve the approximation.

A possibility to reduce the error, is to introduce the effect of the term $R[U]+1$ in the simulation by the insertion of a Metropolis step in the update process, according to the following scheme [3]:

- We start with a configuration $(U, \phi)$; the operator $R[U]+1$ is calculated for the configuration $U$.

- The $\phi$ and $U$ fields are updated $m$ times, by performing a $\phi$ heat-bath together with over-relaxation for the $\phi$ fields and over-relaxation for the $U$ fields.

- The error $R\left[U^{\prime}\right]+1$ is calculated for the new gauge configuration $U^{\prime}$.

- The new configuration $\left(U^{\prime}, \phi^{\prime}\right)$ is accepted with probability

$$
P_{a c c}=\min \left[1, \frac{R\left[U^{\prime}\right]+1}{R[U]+1}\right]
$$


This combination of heat-bath, over-relaxation and Metropolis update ensures that the Lagrangian simulated is the exact QCD one of eq. (9).

The computation of $R[U]+1$ is performed by computing the $Q^{2}$ eigenvalues $\lambda_{i}$ and using:

$$
R[U]+1=\prod_{i=1}^{L} \lambda_{i} P\left(\lambda_{i}\right)
$$

This is a computationally intensive step, because in theory the full spectrum of the matrix $Q^{2}$ has to be determined. In practice, however, when the polynomial is precise enough $\left(\delta(n, \epsilon)<10^{-4}\right)$, the dominant contribution to (10) comes from the low-lying eigenvalues $\lambda<\epsilon$ of $Q^{2}$, and the product (10) can be cut-off at some lower bound $L^{\prime}$. With this method the CPU cost invested for the evaluation of $R[U]$ becomes irrelevant if the Metropolis tests are divided by a sufficient number $m$ of updating steps and the number of eigenvalues $\lambda<\epsilon$ is not excessive. On a $4^{4}$ lattice, possibly thanks to the low density of the eigenvalues, we can choose $\epsilon$ even ten times larger than the lowest eigenvalue, reducing even further the CPU time. Of course, on bigger lattices, $\epsilon$ will have to be chosen closer to the lowest eigenvalue. Notice that the work needed for the global Metropolis test is proportional to $V^{2}$, which renders this algorithm costly for very large lattices.

To obtain the low lying spectrum of $Q^{2}$, we used the Lanczos algorithm. Attention must be paid to roundoff errors, and we chose the method advocated by Cullum and Willoughby to filter out spurious "eigenvalues" [5] (see appendix B).

\section{Numerical simulations with the exact algorithm}

Numerical simulations using the exact algorithm described in the previous paragraph have been performed on a $4^{4}$ lattice in the deconfined phase, at $\beta=6.0$. For the choice of the $(\epsilon, n)$ parameters we have been guided by our experience with the pure Lüscher algorithm. A few exploratory results for a $8^{4}$ lattice, at $\beta=5.3$, and $\kappa=0.156$ and 0.162 (therefore, in the confined phase) will be also presented in the next section, and the related problems discussed.

For the $4^{4}$ lattice, we concentrated on $\kappa=0.14$, where the $\epsilon$ range acceptable for the pure Lüscher algorithm is more limited. We explored the capabilities of the modified algorithm by varying $\epsilon$ and $n$ and compared with previous results at the same parameter values.

The results are listed in Table 2. In the last column of this table, the number of Lanczos iterations performed is reported. The relationship between the number of Lanczos iterations and the actual cut-off $L^{\prime}$ is linear. The values for the average acceptance of the Metropolis step are also shown. Finally, since we chose a value of $m$, the number of $(\phi, U)$ sweeps, equal to or larger than the autocorrelation time $\tau$ (i.e: $m=10$ ), the autocorrelation of the new results in our new units is always $\tau \sim 1$, and is not shown in the table.

In Fig. 4 the plaquette values are plotted vs. $\delta(\epsilon, n)$, along with the corresponding results from the pure Lüscher algorithm. From Table 2 and Fig. 4, it is clear that, when the pure Lüscher algorithm gives satisfactory results, acceptance for the Metropolis step is very 


\begin{tabular}{|c|c|c|c|c|c|c|c|}
\hline$\beta$ & $\kappa$ & $\epsilon$ & $n$ & plaquette & $\delta(n, \epsilon)$ & accep. & $n_{\text {Lan }}$ \\
\hline \multirow[t]{9}{*}{6.0} & \multirow[t]{8}{*}{0.14} & 0.02 & 50 & $0.6082(6)$ & $1.0 \times 10^{-6}$ & 0.97 & 800 \\
\hline & & & 90 & $0.6081(5)$ & $1.0 \times 10^{-11}$ & 0.99 & 800 \\
\hline & & 0.04 & 30 & $0.6093(4)$ & $7.0 \times 10^{-6}$ & 0.89 & 800 \\
\hline & & & 60 & $0.6084(6)$ & $3.6 \times 10^{-11}$ & 0.98 & 800 \\
\hline & & \multirow{4}{*}{0.08} & 90 & $0.6094(5)$ & $1.9 \times 10^{-16}$ & 0.98 & 800 \\
\hline & & & 16 & $0.6072(5)$ & $1.0 \times 10^{-4}$ & 0.71 & 300 \\
\hline & & & & $0.6083(5)$ & $1.0 \times 10^{-4}$ & 0.69 & 1000 \\
\hline & & & & $0.6088(6)$ & $1.0 \times 10^{-4}$ & 0.71 & 2000 \\
\hline & HMC & & & $0.6094(2)$ & & & \\
\hline
\end{tabular}

Table 2: Simulation results for the plaquette on a $4^{4}$ lattice at $\beta=6$ for the exact algorithm (Lüscher+Metropolis) and the HMC algorithm. Each plaquette value is an average of about $10^{\prime} 000$ measurements.

high, and no significant differences are observed when using the exact algorithm. Where the approximation of the pure Lüscher algorithm breaks down (in Table 2, for parameters $\epsilon=0.08, n=16)$, on the other hand, the average acceptance goes down to about $70 \%$, and a significant improvement in the plaquette value is observed, meaning that the insertion of the $R[U]+1$ term actually corrects the error the approximation makes for $s<\epsilon$. This is clearly illustrated in Fig. 5, where the plaquette value is shown to improve as more and more terms in the product $\prod_{i=1}^{L} \lambda_{i} P\left(\lambda_{i}\right)$ are computed.

These results show that the exact algorithm can be effectively used to perform computations at higher values of $\epsilon$ or lower values of $n$ than acceptable for the pure Lüscher algorithm, thus reducing the CPU cost of the simulation.

As for the scaling behaviour, that of the original version of the algorithm is $V(\log V)^{2}$. The version of the algorithm with the global Metropolis test introduces additional work for the evaluation of the low spectrum of the fermion matrix. Its scaling behaviour is more critical: $a V(\log V)^{2}+b V^{2}$, where $a \gg b$ are prefactors.

\section{Numerical simulation on the $8^{4}$ lattice}

We finally present here a few results obtained on an $8^{4}$ lattice with the aim of exploring the behaviour of the pure Lüscher and exact algorithm in a situation nearer to the physical one, i.e. on a larger lattice in the confined phase, and with relatively light quarks. The data taken are not exhaustive, and we only intend to sketch the possible problems inherent to simulations with such parameters, and show the indications coming from a few numerical results.

The main problem we must be ready to encounter has already been mentioned: on an $8^{4}$ lattice, the density of the $Q^{2}$ eigenvalues is higher than on a $4^{4}$ one. This means, on the 


\begin{tabular}{|c|l|l|l|l|l|l|l|l|l|}
\hline $\mathrm{k}$ & $\epsilon$ & $n$ & plaquette & $W(1,2)$ & $W(1,3)$ & $W(2,2)$ & $\chi(2,2)$ & accept. & $n_{\text {Lan }}$ \\
\hline \hline \multirow{3}{*}{0.156} & \multirow{3}{*}{0.007} & 80 & $0.485(1)$ & $0.248(1)$ & $0.128(1)$ & $0.071(1)$ & $0.56(3)$ & & \\
& & 80 & $0.483(1)$ & $0.248(3)$ & $0.127(3)$ & $0.071(2)$ & $0.56(3)$ & 0.88 & 2000 \\
& & & $0.4847(3)$ & $0.2472(3)$ & $0.1274(3)$ & $0.0712(2)$ & $0.571(8)$ & & HMC \\
0.162 & \multirow{2}{*}{0.01} & 60 & $0.508(1)$ & $0.278(2)$ & $0.153(2)$ & $0.096(2)$ & $0.46(1)$ & & none \\
& & 60 & $0.5057(6)$ & $0.2740(5)$ & $0.1500(4)$ & $0.0914(2)$ & $0.485(6)$ & 0.80 & 1000 \\
& & 60 & $0.5023(6)$ & $0.2686(5)$ & $0.1461(5)$ & $0.0876(2)$ & $0.494(6)$ & 0.72 & 2000 \\
& & & $0.5016(4)$ & $0.2679(5)$ & $0.1451(4)$ & $0.0870(4)$ & $0.497(6)$ & & HMC \\
\hline
\end{tabular}

Table 3: Simulation results for the plaquette on a $8^{4}$ lattice at $\beta=5.3$ for the Lüscher+Metropolis algorithm and the HMC algorithm. Each plaquette value is an average of about $4^{\prime} 000$ measurements. Here $W(I, J)$ represents the $I \times J$ Wilson loop, and $\chi(2,2)$ is the $(2,2)$ Creutz ratio.

one hand, that we will have to use an $\epsilon$ value much closer to the minimum eigenvalue than in the $4^{4}$ case, and on the other hand that, when the global Metropolis test is introduced, a very large number of Lanczos iterations will be necessary to determine all eigenvalues up to $\epsilon$.

Let us now look, in Table 3 and Fig. 6 , at the results of the simulations performed at $\beta=5.3, \kappa=0.156$ with $\epsilon=0.007, n=80\left(\delta=3 \times 10^{-6}\right.$, lowest eigenvalue $\left.\sim 0.001\right)$, and $\kappa=0.162$ with $\epsilon=0.01, n=60\left(\delta=1 \times 10^{-6}\right.$, lowest eigenvalue $\left.\sim 0.0005\right)$, both without and with the global Metropolis test. We chose the $\beta$ and $\kappa$ values such as to reproduce HMC results by Gupta et al. [6], which are also reported in Table 3, for comparison.

Both the $(\epsilon, n)$ range explored and the statistics are too poor to allow us to draw definitive conclusions; nevertheless, we can derive from these data some indications. For $\kappa=0.156$, where we used $\epsilon=0.007$, with a lowest eigenvalue $\sim 0.001$, we can see that the pure Lüscher algorithm gives quite good results, and the global Metropolis test has little influence (corresponding to an acceptance rate of $88 \%$ ).

When we go to $\kappa=0.162$, with a lowest eigenvalue $\sim 0.0005$, and simulate at $\epsilon=0.01$ (20 times larger), the Lüscher algorithm result differs markedly from the HMC one; we now introduce the global Metropolis test, and we see that it brings the result to agreement with HMC, within the statistical error.

One apparently puzzling observation is that all plaquette and Wilson loop values converge to the HMC ones from above, whereas, since the quenched results are lower than the full QCD ones, one might have expected a monotonic convergence from below. To investigate this phenomenon, we combine the Wilson loop results to form the $(2,2)$ Creutz ratio $\chi(2,2)$

$$
\chi(2,2)=-\log \frac{W(2,2) W(1,1)}{W(1,2)^{2}}
$$


which has a physical significance, since it approximates $(\sigma a)^{2}$, the squared string tension in lattice units. From Table 3 and Fig. 7 we see that this quantity converges to the correct limit from below, whereas the quenched value is above the full QCD one. Thus monotonic convergence is not guaranteed, even for physical observables. This will make extrapolation to the exact results a rather delicate matter.

From the analysis of these data, we have confirmation of previous results on the accuracy of the approximation and also of the expected CPU requirements at larger volume.

\section{Conclusion}

We have presented first results of full QCD simulations using Lüscher's method. Measurements of the plaquette on a $4^{4}$ lattice show that the approximation remains quite accurate for that observable, even when the cutoff parameter $\epsilon$ is increased far above the lowest

eigenvalue $\lambda_{\min }\left(Q^{2}\right)$. They also support our analysis of the complexity of the algorithm: $V(\log V)^{2}$ with the volume $V$ of the system, $m_{q}^{-3}$ or better with the quark mass $m_{q}$. This compares favorably with Hybrid Monte Carlo $\left(V^{5 / 4}\right.$ and $\left.m_{q}^{-13 / 4}\right)$.

Larger Wilson loop measurements on an $8^{4}$ lattice reveal the systematic errors of the method. Unlike most approximations to dynamical quarks, it turns out that the approach of Wilson loops and Creutz ratios to their correct values is not monotonic as the approximation improves.

We have tested a variant of Lüscher's algorithm, which includes a Metropolis step to correct for the breakdown of the approximation for small eigenvalues. This extra step brings Wilson loops to their correct values within errors, for an overhead which is small on our lattice sizes, but grows like $V^{2}$. Further variants are being explored, in particular an exact non-hermitian one proposed in [8].

\section{Acknowledgements}

We acknowledge helpful suggestions from A.Boriçi; we thank R.Sommer and B.Jegerlehner for useful discussions, and P.Arbenz and M.Gutknecht for insights on the Lanczos algorithm. Our thanks also go to all authors of reference [2], for allowing us access to parts of their software and documentations. Finally, we thank the SIC of the EPFL for granting us access to the computer resources of the EPFL. 


\section{Appendix}

\section{A Implementation}

The program is written in FORTRAN 77 for the Cray-T3D using communication primitives of the Cray. Its structure is organised in four levels and a user interface facilitates multiuser manipulations. From the bottom up, the first level controls communication, navigation (see below) and memory allocation between the processors (PE's). The second level constructs all objects needed in the simulations (for example staples, plaquettes, $Q$ and $Q^{2}$ vector multiplications,...). The third level contains all the algorithms used for the simulation. The fourth level is the envelope which controls the whole program. A common user interface to facilitate compilation and input/output is defined.

The lattice $\Lambda$ is first divided into hypercubes of side 2 (containing $2^{4}$ points each), then adjacent hypercubes are grouped into $n p$ 円 equal sets $\Lambda_{i} \subset \Lambda \quad(i=0, \ldots, n p-1)$, each of which will be assigned to one PE. For the navigation in the lattice a mapping Navig $: \Lambda \rightarrow \Lambda_{i}$ between the physical coordinates on the lattice and their allocation as local sublattice coordinates is stored as a table on each PE. For a given lattice coordinate $x \in \Lambda$ the mapping Navig returns the corresponding local coordinate $x_{i} \in \Lambda_{i}$ and the identification number $i$ of the PE which owns $x_{i}$. The memory allocation of gauge and bosons fields is organised according to this mapping. The communication of data is also controlled by this mapping.

All objects are constructed in parallel by the PE's which locally operate on their sublattices. The construction of the objects needs some communication of data between PE's. The communication is performed asynchronously using the shmem_get/shmem_put routines of the Cray-T3D. Only at the algorithmic level the needed synchronisations are defined.

All algorithms used (heat-bath, over-relaxation, global Metropolis, Lanczos) are constructed using as input the objects. The heat-bath and over-relaxation algorithms for the boson fields are specifically written for the Lüscher local bosonic action. All other algorithms are general and can be also used for quenched simulations by simply switching some libraries at level 2.

\section{B Lanczos process}

The Lanczos algorithm is a powerful technique used for the determination of the spectrum of sparse, symmetric matrices[7]. During the iterations of the Lanczos algorithm a $m \times m$ tridiagonal symmetric matrix $T^{(m)}$ is obtained by transforming a $n \times n$ matrix $A$ (with $m \ll$ $n$ ) with a matrix $Q$ whose columns are called Lanczos vectors. The extremal eigenvalues of $T^{(m)}$ are estimates of the extremal eigenvalues of $A$.

\footnotetext{
${ }^{1}$ Here $n p$ represents the number of used PE's. On the Cray-T3D only $n p$ equal to a power of 2 is allowed.
} 
In exact arithmetic, the Lanczos vectors are orthogonal. However, in finite precision arithmetic, they lose mutual orthogonality as the number of iteration steps $m$ increases. As a consequence eigenvalues re-appear, i.e. the algorithm finds "spurious" or "ghost" values which are not actual eigenvalues of $A$, but which are nearly degenerate with them.

The problem can be solved by explicitly orthogonalizing the Lanczos vectors. However, all the Lanczos vectors must then be stored and the memory cost of the algorithm is enormous.

Instead we use another clever way of identifying spurious eigenvalues without orthogonalization, proposed by Cullum and Willoughby [5]. In their algorithm one the eigenvalues of $T^{(m)}$ are compared with the eigenvalues of a matrix $T_{2}$ which is obtained from $T^{(m)}$ by deleting its first row and column. If a simple eigenvalue of $T^{(m)}$ is also a simple eigenvalue of $T_{2}$, then this eigenvalue is spurious. 


\section{References}

[1] S.Duane, A.Kennedy, B.Pendleton, and D.Roweth, Phys. Lett. B 195, 216 (1987)

[2] M.Lüscher, Nucl. Phys. B 418 (1994) 637

B.Bunk, K.Jansen, B.Jegerlehner, M.Lüscher, H.Simma and R.Sommer , to appear in Nucl. Phys. B (Proc. Suppl) 42 (1995)

[3] M.Peardon, to appear in Nucl. Phys. B (Proc. Suppl) 42 (1995).

[4] M.Creutz, Phys. Rev. D 38 (1988) 1228

R.Gupta, G.Kilcup and S.Sharpe, Phys. Rev. D 38 (1988) 1278; 38 (1988) 1288

[5] J.Cullum and R.A Willoughby, J. Comp. Phys., 44 (1981) 329

[6] R.Gupta et al. , Phys.Rev. D 40 (1989) 2072

[7] G.H. Golub and C.F. v. Loan, Matrix computations, secon edition, (The Johns Hopkins University Press, Baltimore, 1990)

B.N. Parlett, The symmetric eigenvalue problem, Prentice-Hall Series in Computational Mathematics, (Prentice-Hall, N.J., 1980)

I.M.Barbour et al. The Lanczos method in lattice gauge theory, in: The recursion method and its applications, Springer Series in Solid-State Sciences 58, eds. D.G.Pettifor and D.L.Weaire (Springer-Verlag, Berlin, 1985)

[8] A. Boriçi and Ph. de Forcrand, IPS Report 95-13, hep-lat 9505021 


\section{Figure caption}

1. Lower spectrum of the $Q^{2}$ matrix at $\beta=6$ and $\kappa=0.12$ on a $4^{4}$ lattice.

2. Plaquette versus $\delta$ at $\beta=6$, (a) $\kappa=0.12$ and (b) $\kappa=0.14$ on a $4^{4}$ lattice with the pure Lüscher algorithm. The lines indicate the HMC values, with their errors.

3. Autocorrelation time of the plaquette versus $n / \sqrt{\epsilon}$ for a $4^{4}$ lattice, at $\beta=6$., (a) $\kappa=0.12$ and (b) $\kappa=0.14$. The lines represent linear fits to the data.

4. Plaquette versus $\delta$ at $\beta=6, \kappa=0.14$ on a $4^{4}$ lattice with the use of the global Metropolis test. The lines indicate the HMC values, with their errors.

5. Plaquette versus number of Lanczos iterations used in the global Metropolis test, at $\beta=6, \kappa=0.14$ on a $4^{4}$ lattice, with parameters $\epsilon=0.08$ and $n=16$. The lines indicate the HMC value, with its error.

6. Plaquette (a) and Wilson loop $W L(1,2)$ (b) data from the simulation on the $8^{4}$ lattice at $\beta=5.3$ with $\kappa=0.156(\diamond)$ and $\kappa=0.162(\square)$. The lines indicate the HMC values, with their errors.

7. Creutz ratio $\chi(2,2)$ versus number of Lanczos iterations used in the global Metropolis test, on the $8^{4}$ lattice, $\beta=5.3, \kappa=0.162, \epsilon=0.01$ and $n=60$. The lines indicate the HMC value, with its error. 


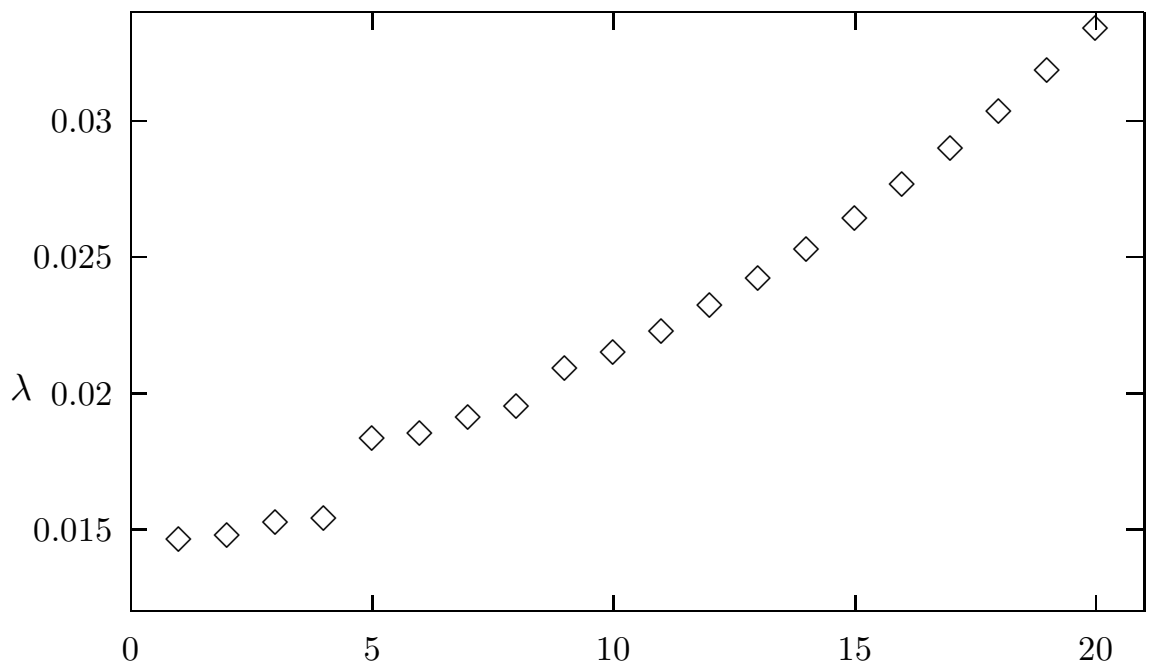

Eigenvalue number

Figure 1 


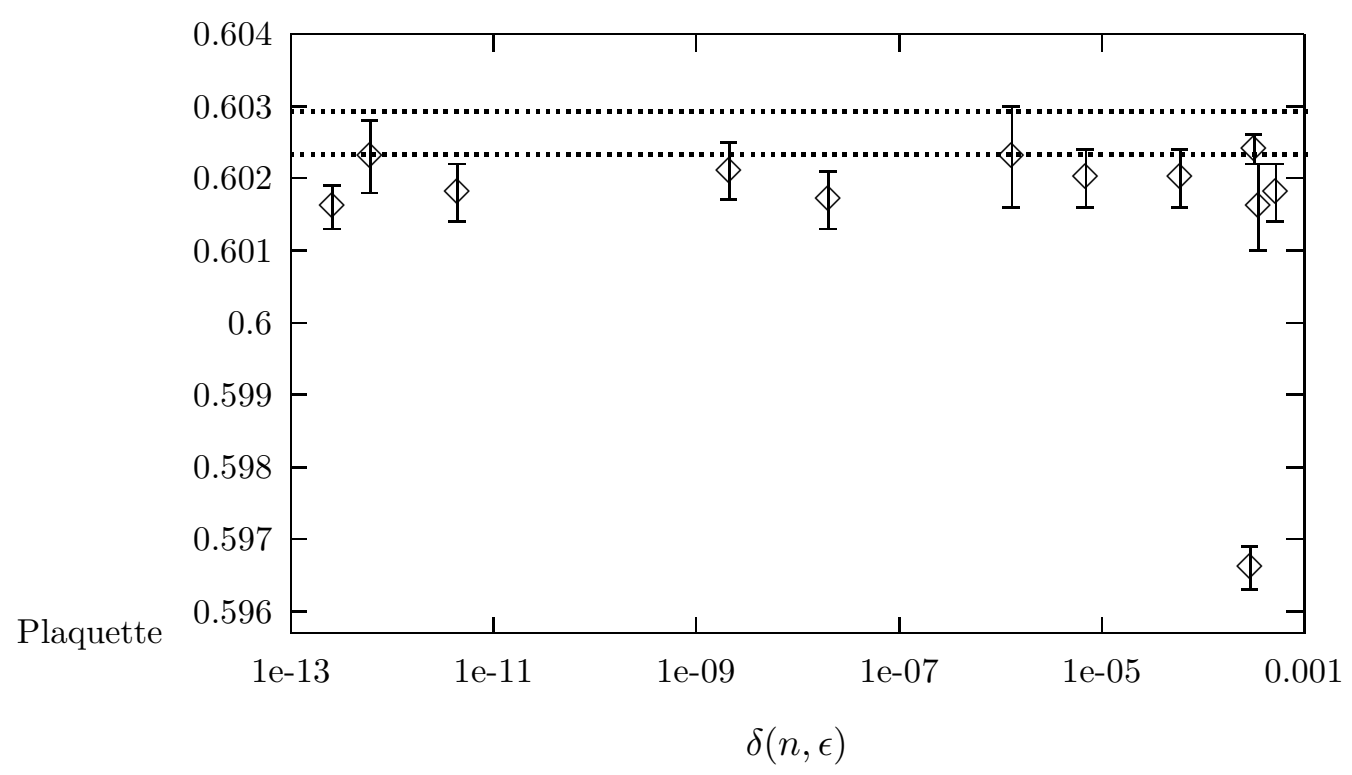

Figure 2a

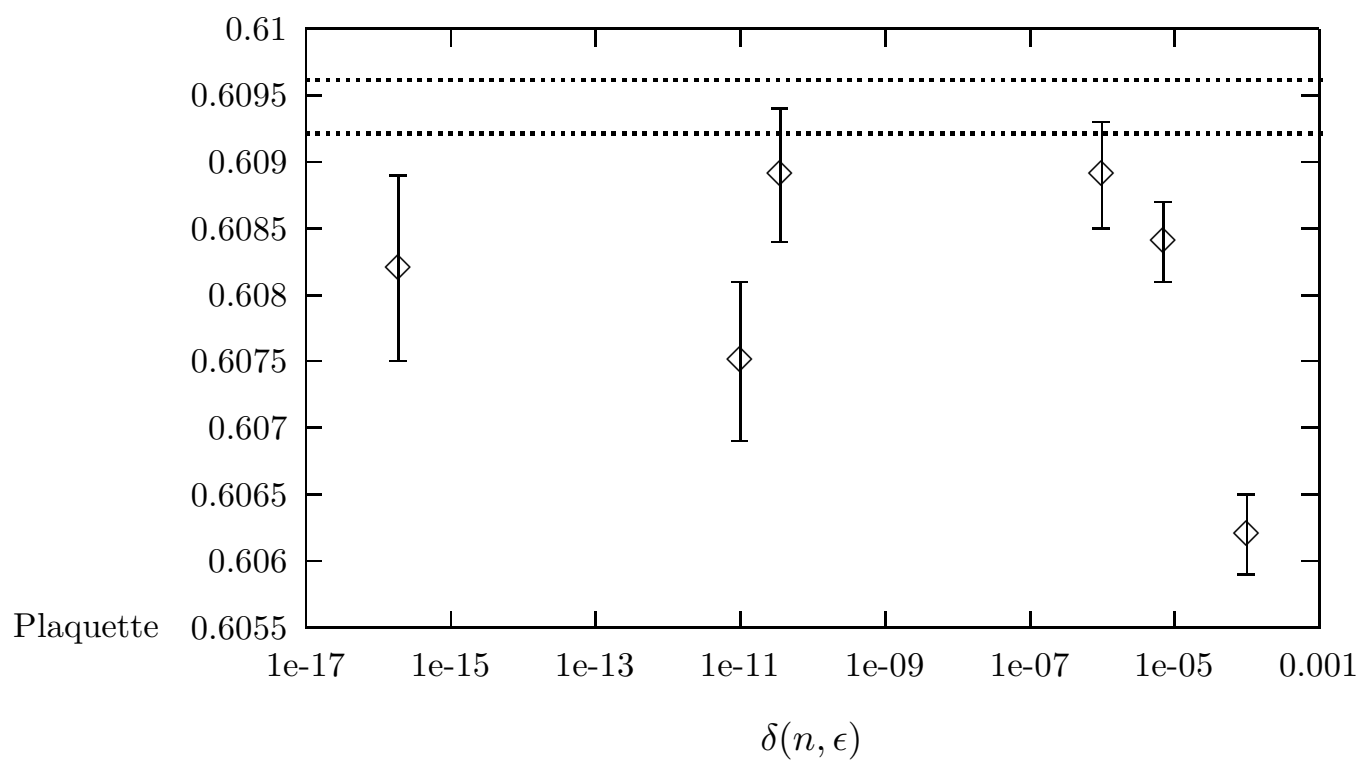

Figure $2 b$ 


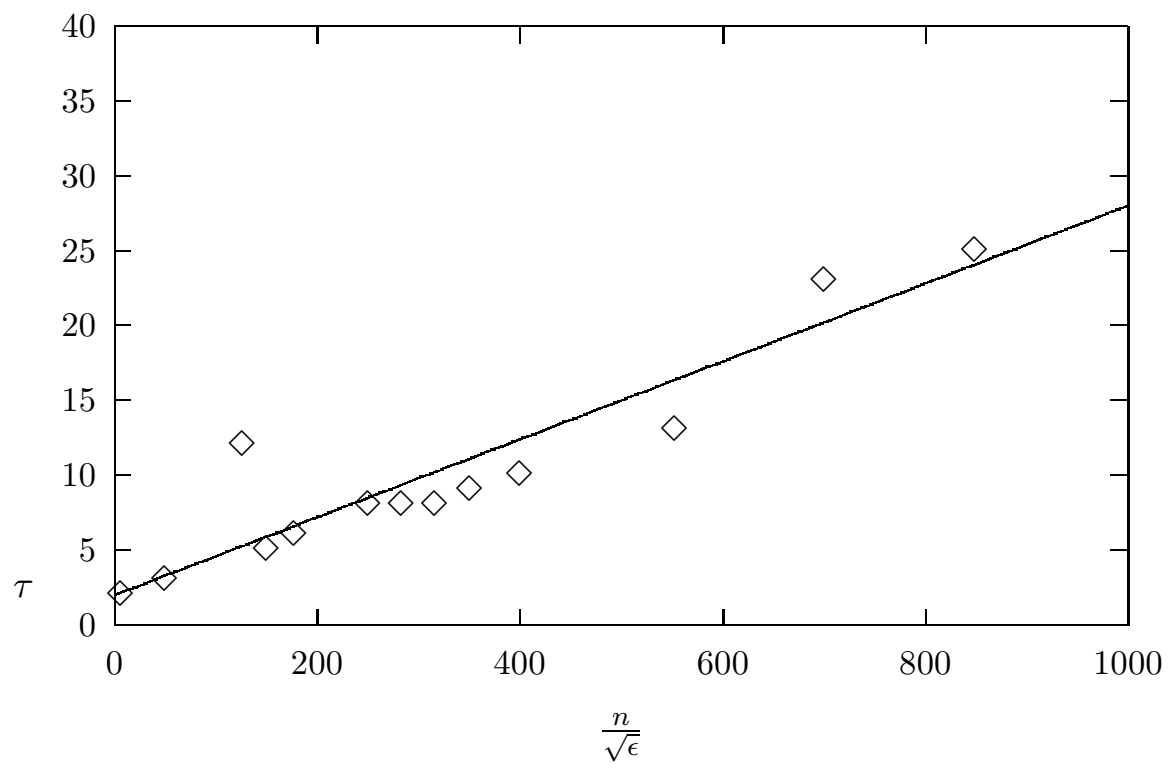

Figure $3 \mathrm{a}$

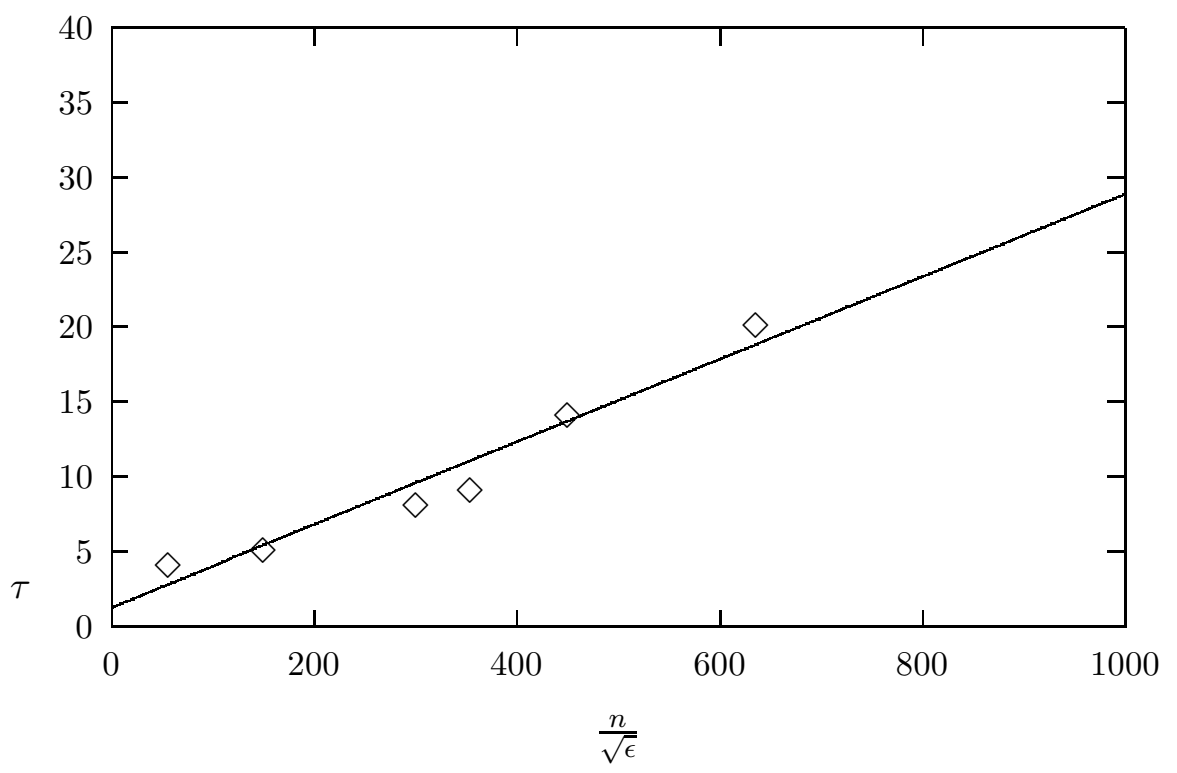

Figure 3b 


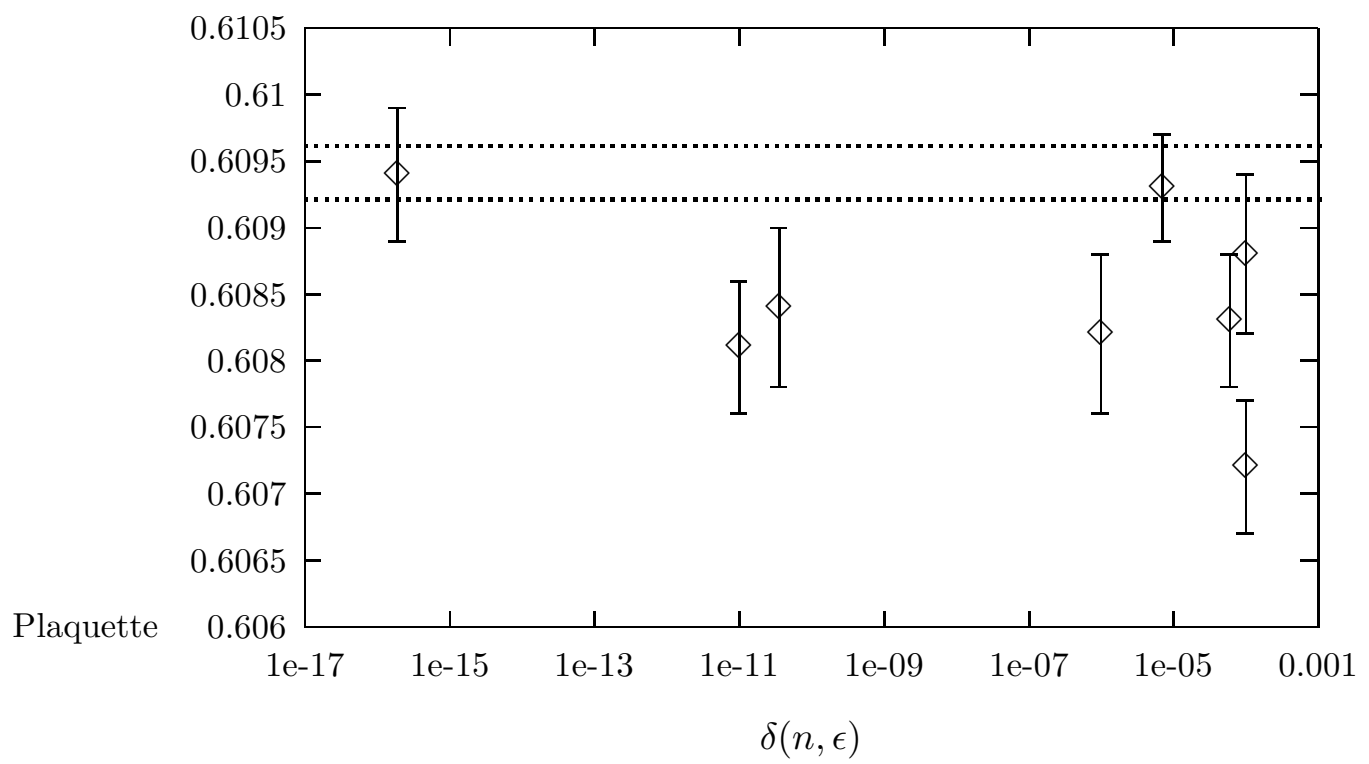

Figure 4 


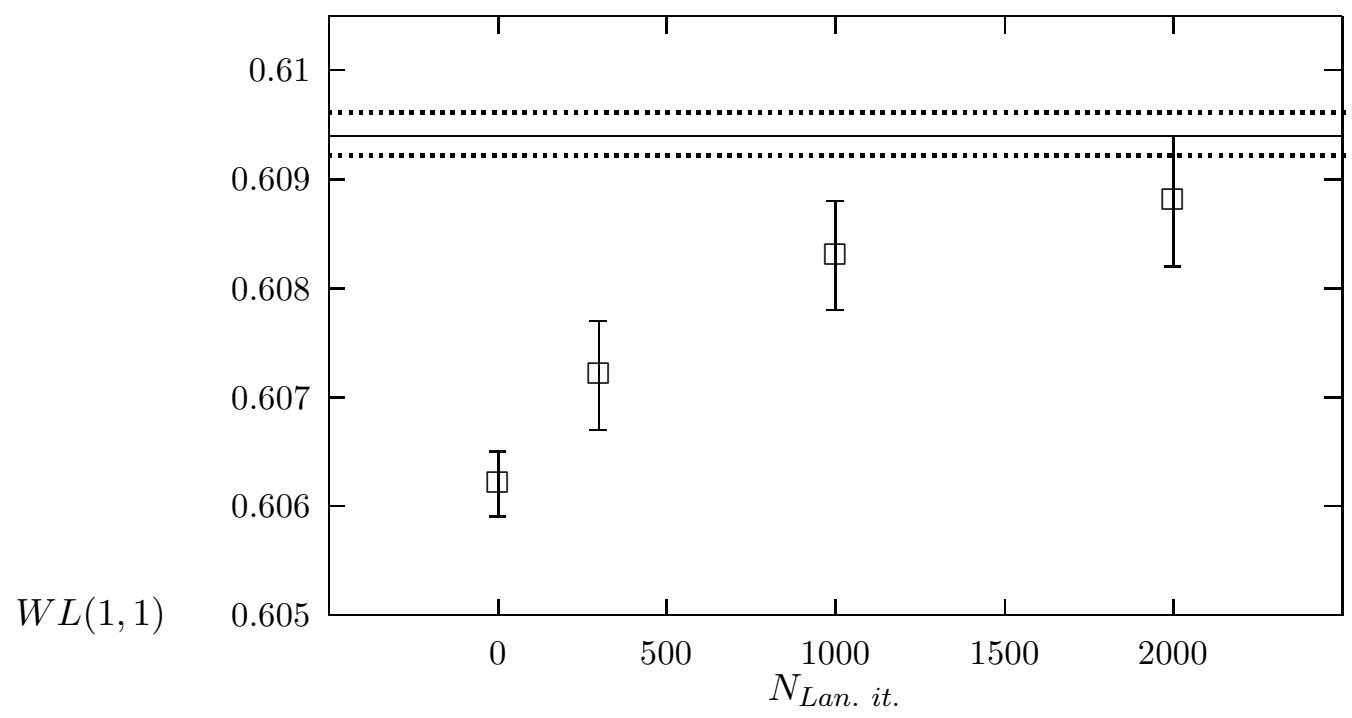

Figure 5 


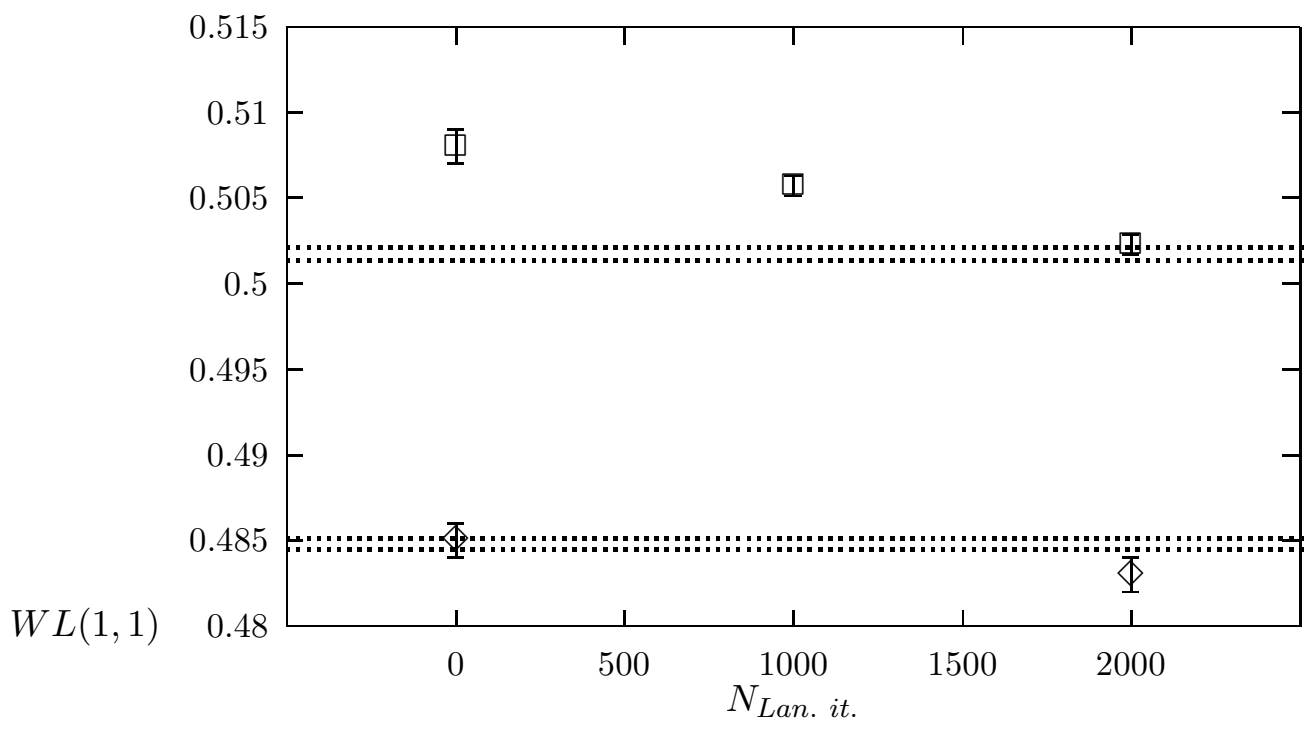

Figure 6a

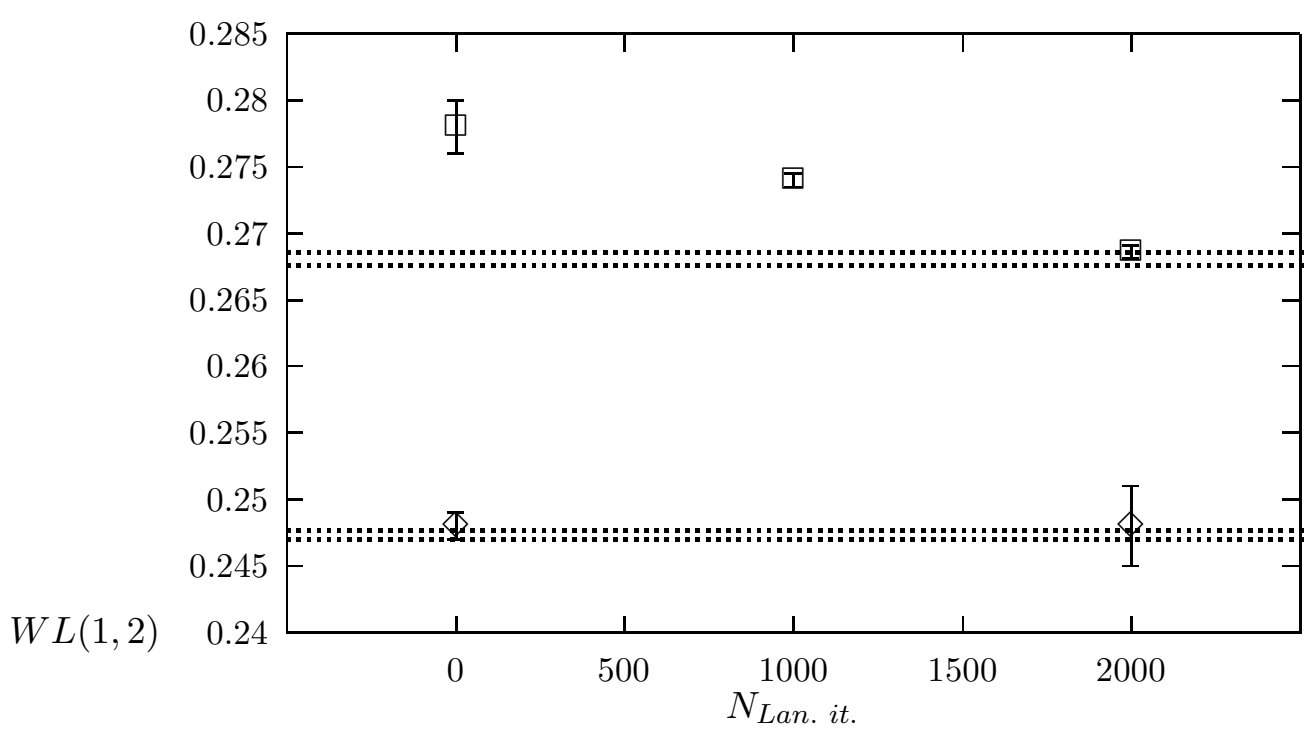

Figure $6 \mathrm{~b}$ 


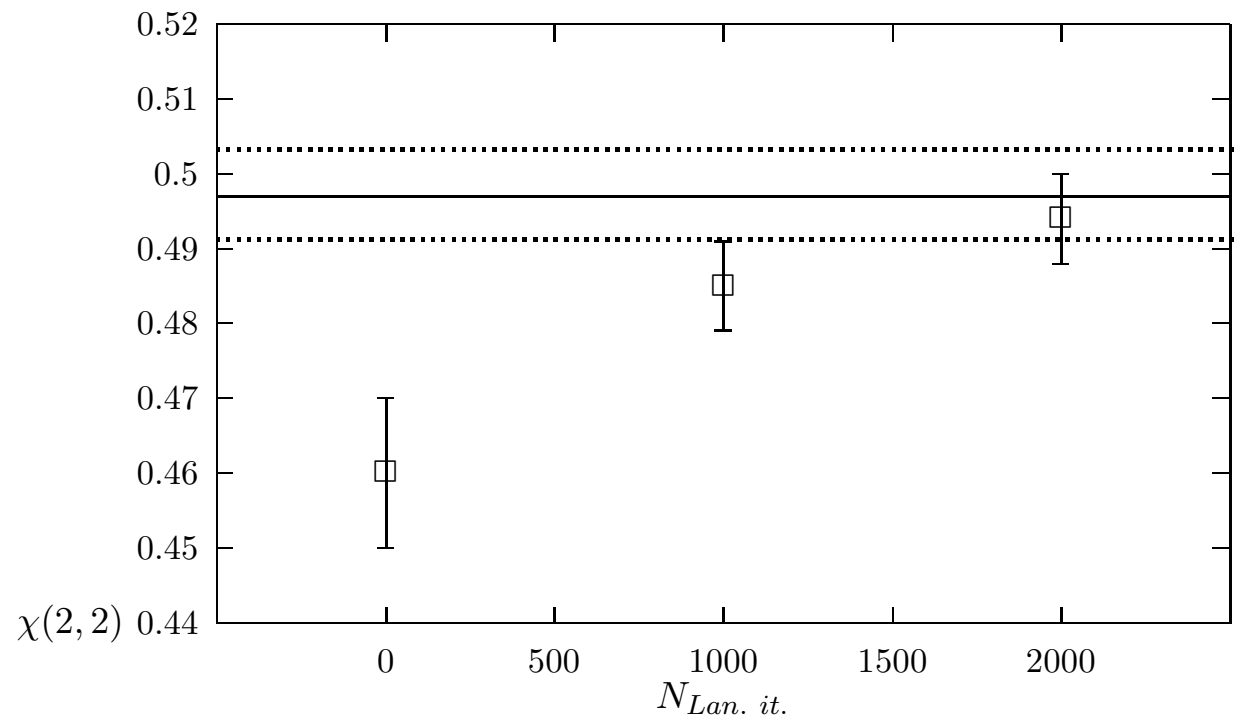

Figure 7 